\title{
Immersive Storytelling for Information Security Awareness Training in Virtual Reality
}

\author{
Philipp Ulsamer, Andreas E. Schütz, Tobias Fertig, and Lisa Keller \\ Faculty of Computer Science and Business Information Systems \\ University of Applied Sciences Würzburg-Schweinfurt \\ Sanderheinrichsleitenweg 20, 97074 Würzburg, Germany \\ \{philipp.ulsamer, andreas.schuetz, tobias.fertig, lisa.keller\}@fhws.de
}

\begin{abstract}
Due to the central role of the human factor in information security, the need for information security awareness (ISA) is constantly increasing. In order to maintain a high level of ISA, trainings have to be carried out frequently to ensure sustainability. Since education via virtual reality (VR) has led to a sustainable learning effect in other fields, we evaluated the use of VR for ISA trainings. Moreover, we combined our VR training with immersive storytelling. For the evaluation we used two sets of participants. The first used a traditional e-Learning method to answer the questionnaire. The second used our VR training. After one week we repeated the questionnaires. The results showed that the VR group could achieve higher scores than the noVR group. Moreover, the VR group achieved even higher scores after one week which might be due to the sustained learning effect from the VR training.
\end{abstract}

\section{Introduction}

The human factor plays a major role in the information security of institutions. The behavior of employees at their workplaces and at home affects the three safety objectives confidentiality, integrity, and availability of corporate data. Vulnerability can, for example, occur by sharing confidential PIN codes, leaving confidential documents on desks, or using foreign USB devices. These risks and many others can be minimized by increasing the employee's ISA of potential dangers.

The ease of exploiting the "human factor" as a weak point leads to an increasing number of attacks with techniques such as phishing, malware, and social engineering [1]. Former social engineer Kevin Mitnick puts it this way [2]: "Cracking the human firewall is often easy, requires no investment beyond the cost of a phone call, and involves minimal risk."

To make employees aware of the importance of their role, companies typically carry out ISA campaigns.
However, those campaigns or trainings should be repeated frequently in order to remain a high level of ISA and to stay in the employee's mind [3]. Within this research project our goal is to create a sustainable learning environment. Since it has been proven a number of times that VR is an efficient tool to achieve sustainability [4], we evaluated the use of VR for ISA training. The efforts of organizing repeated trainings could be decreased through a more sustainable approach.

VR can also be beneficial in case of time problems, physical inaccessibility, limits due to a dangerous situation as well as ethic problems according to [5]. Regarding ethic problems, VR is already used to successfully train serious surgeries with non-experts [6]. Since it is not ethical to actually infiltrate an institution by using social engineering, VR can be a beneficial tool to increase the ISA of employees.

For this research project we will be using a 360-degree VR video, which might lead to some challenges regarding the attention of the viewer: Our participants are able to turn in any direction at their free will and possibly miss important parts of the plot. To avoid loosing the participants' attention and to ensure high immersion, we create a digital story including actors. According to [7] the use of actors and immersive storytelling were efficient tools to guide the attention of their participants. Moreover, we are hoping that the immersive storytelling transferred by our actors will increase the sustainability of the learning experience.

In the following we begin by defining our two research questions as well as our two hypotheses. Afterwards, we summarize related work. Here, we cover three different topics: ISA, learning environments with VR, as well as storytelling in VR. In Section "Research Approach" we describe our approach of the conducted study. In Section "Study Population and Sampling", we present the demographics of the participants of this study. Following the summary of used data sources, we gather and analyze the research results. The results are discussed in Section "Results Discussion", where we 
will also answer our research questions. Finally, we will give a short summary of the paper and will conclude with an outlook and future work.

\section{Research Questions and Hypotheses}

The aim of this paper is to increase immersion through storytelling by including actors playing roles of characters in a predefined story structure. Actors will guide users' attention, and therefore, prevent the viewer to experience motion sickness. This should not only increase the edutainment factor of an ISA training, but also manifest the acquired knowledge longer than in traditional e-Learning methods. The following research questions and hypotheses will be investigated within this paper:

RQ1. Can an increased knowledge in the field of social engineering be achieved through immersive storytelling in VR as opposed to traditional e-Learnings?

RQ2. Does immersive storytelling in VR have a more sustainable learning outcome than traditional e-Learnings?

$H_{0}$ If subjects use the traditional e-learning, they gain an equal or higher score in the ISA test compared to subjects who used the VR environment.

$H_{1}$ If subjects use the VR environment, they gain a higher score in the ISA test compared to subjects who use the traditional e-learning.

\section{Related Work}

The study of storytelling for ISA in VR requires the understanding of multiple topics: general e-Learning approaches in the field of ISA, the use of VR in learning environments, as well as the concept of storytelling in virtual worlds. Since the learning objective of the participants focuses on social engineering, this attack method will be examined more closely in this paragraph.

\subsection{Social Engineering}

Social engineering is a very realistic threat for companies. The most prominent and successful social engineering technique is phishing [8]. The attackers manipulate the victims to take an action, that may not be in the company's best interest [9]. A social engineer may try to obtain login credentials by pretending to be an IT administrator and simply ask users over the phone to share their password. This social engineering technique is called pretexting or impersonation [10, 11].
In the social engineering attack cycle of Kevin Mitnick [2] a social engineer passes four phases: research, developing rapport and trust, exploiting trust, and utilizing information.

\subsection{Information Security Awareness in Learning Environments}

Attacks by cyber criminals on users of information systems are constantly increasing [12, 13]. In such attacks, it is often easier for attackers to exploit the human factor than to look for technical weaknesses in order to hack a system in the classic way, such as brute-forcing. Social engineering is, besides the distribution of malware, a popular method for user-centered attacks [1]. To counter these threats, ISA has been established as a separate research area within information security. ISA addresses how users can be convinced to behave in accordance with information security policies, guidelines and best practices. Information security compliant behavior is the result of a person's knowledge, habit, salience, and behavioral intention in relation to a particular behavior [14]. High ISA minimizes the risk of users being exploited as weak spots in the information security concept. Especially in companies, ISA campaigns are utilized to sensitize employees towards the importance of their role in securing their companies information assets. The use of learning environments, such as e-Learnings, is a popular method within such campaigns.

Schütz et al. [15] describe e-Learning as a suitable tool to increase the ISA of users. The authors show that e-Learning can help especially when wanting to influence the knowledge and habits of a user. The salience and intention, however, can only be influenced in combination with other measures, such as social marketing or discussion groups. Especially due to human emotions and beliefs, e-Learnings can also have negative outcomes. The authors do not differentiate between different forms of e-Learnings, like textual based e-Learning or e-Learning via Virtual Reality. They also give only an exploratory point of view with no concrete underlying qualitative or quantitative measurements.

However, according to Fertig et al. [16] a common approach to measure the success of e-Learnings is to run surveys and questionnaires with the participants. Since the manual evaluation of those surveys and questionnaires is a time-consuming task, the authors proposed an approach to automate the measuring. They derived metrics for success and effectiveness but recognized that success can hardly be measured 
automatically. Since we are not conducting our study within an institution it is impractical to implement automated measurements. Therefore, we will evaluate our surveys manually instead of using the proposed metrics of Fertig et al.

\subsection{Learning Environments with Virtual Reality}

A great amount of research covers the increasing motivation of the user in physical [17] and theoretical [18, 19] learning fields through virtual environments. The traditional e-Learning approach is difficult to apply to educational topics that are difficult to learn [18]. VR makes up for the shortcomings in e-Learning and can help to simplify the teaching of scientific visualizations, situational awareness and monitoring of complex technical objects [20]. The use of VR in learning environments also offers many advantages. VR allows the experience of events that are not physically reachable. Learning and training environments can be created in safe areas to avoid potential dangers [5]. To completely immerse into a virtual environment tools are required that used to be expensive but have now become more affordable for the masses. A disadvantage is the usability of the software and the interface of the device. The fact that you can dive into another world within seconds and perform movements in this world that do not take place in the real world can lead to motion sickness. In addition, the user has the freedom to explore the virtual world in VR and could experience a so called fear of missing out due to the abundance of images [21, 22]. Therefore, we will use storytelling and the use of actors to draw attention to important learning content while at the same time offering the user the opportunity to explore the VR world and become part of it.

Huang et. al [23] present constructive teaching approaches that could help educators in the development of virtual reality learning environments (VRLE) which allow a more efficient use. They state that the major difficulty is the navigation in a virtual environment, where the user can easily feel lost. Poor usability also limits the effectiveness of teaching content to the user and causes motion sickness when there is no orientation in the virtual world [24]. Furthermore, challenges mentioned by the authors are the development of VR courses which require a certain skill level as well as the cost factor when purchasing VR hardware. Finally, they state that the comparison between the effectiveness of 3D and 2D environments is not addressed in their research.

\subsection{Storytelling in Virtual Reality}

Immersion is divided into two categories: Technical and Narrative Immersion [25]. Technical immersion is achieved by the viewer perceiving himself as part of the virtual environment [26] and being recognized by actors as part of the virtual world [27]. In this category, diegetic methods, such as lights and sounds [27, 28, 29, 30] and non-diegetic methods, such as arrows and forced rotation [31, 32] are used in attention guidance to allow the viewer to explore the virtual world and to follow the story in an omnidirectional virtual environment.

The narrative immersion refers to the influence that the story content and structure have on the viewer. Ryan [33] distinguishes between four dimensions of narrative immersion in VR: spatial, temporal, spatio-temporal, as well as emotional immersion. Spatial immersion focuses on the setting, place and time of the story which gives the viewer the feeling of being in the virtual place. Temporal immersion focuses on the structure and generation of a plot like character-driven or plot-based storytelling [34]. Spatio-temporal immersion is affected by the narrative position as well as the absorption of the audience within the story. Emotional immersion deals with the recipients emotions that the story elicits. Elmenzeny et. al [25] applied immersive storytelling in a 360-degree video setting to analyze the interplay between narrative and technical immersion. They came to the conclusion that the basis for immersion is spatial, which is supported by the other narrative types. Narrative and technical aspects can be combined to tell the story in a certain style and with an increased immersion. Emotional immersion for example can be achieved through suspense created by the structure of the story. The level of immersion can be regulated by visual cues like lighting effects. As a limitation of their work, the authors note that the 360-degree videos researched, although originating from different genres, are driven by narratives. Cues are important to guide the viewer's attention in a desired direction. However, it can easily be overlooked by the user, as first time watchers are busy exploring the virtual world, especially for videos which do not have narrative content.

Rizvic et. al [7] involved actors in VR digital stories to achieve a higher level of user immersion. The acting of characters inhabiting cultural monuments increased VR environments emotions and empathy of users. Their study showed that the use of actors in VR storytelling improves the edutainment value of virtual cultural heritage applications. They also dealt with the limitations mentioned by Elmenzeny et. al [25] when facing attention guidance in 360-degree videos. They 


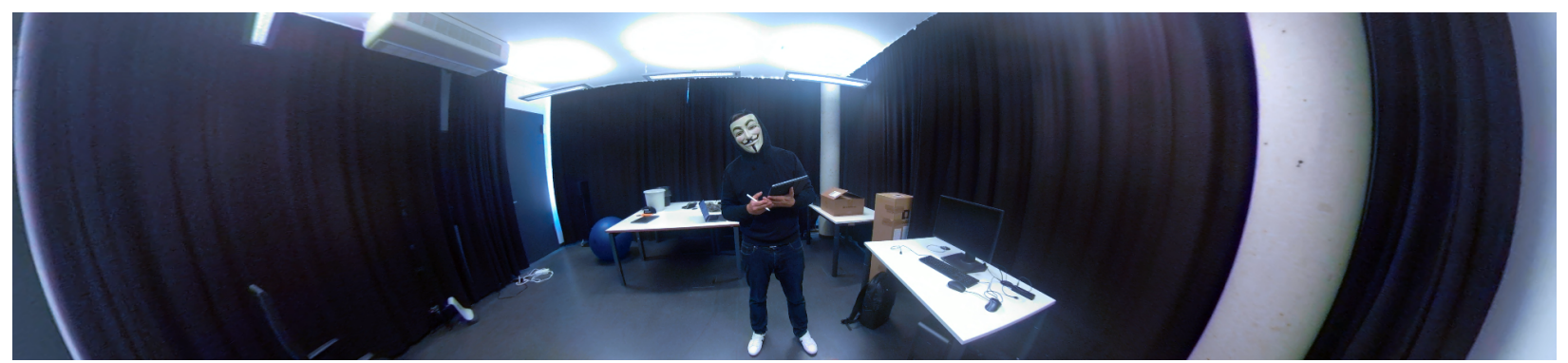

Figure 1. Actor in immersive storytelling in VR.

found out that the viewer's attention was always directed to the speaking actor no matter which Point-of-View (PoV) the user had before, even when the user explored the historical view for the first time. This was also supported by the fact that with several actors, the speaking actor was highlighted by visual cues. Despite the rising ISA campaigns in companies around the importance neither to reveal nor negligently to deal with company information, the human factor is still regarded as the biggest weak point in information security. By using immersive storytelling in VR, the sustainability of the taught knowledge in ISA training should be significantly increased in contrast to traditional plain text-based e-learning methods, which is a missing topic in current literature.

\section{Research Approach}

For our experiment, we used a 2x2 between-subject design. We have distinguished between two groups. One group, which is called noVR group in the following, has been given an plain text-based e-Learning platform as learning content. The other group, subsequently called VR group, was given an environment in a virtual world that represented the same content. Each group was tested twice at intervals of one week.

Participants of the noVR group were first invited to read the instructions for the ISA test through the Lime Survey tool and received an introduction to the experiment. Subsequently, socio-demographic data such as age, gender, school education, experience in ISA and their background in STEM (science, technology, engineering, mathematics) were collected from each participant. Whether participants have a background in STEM or not was covered in order to estimate how familiar they might be with technology as well as ISA which could influence their ISA test results. After the subject had received an e-Learning presentation about the content, each subject was asked ten questions about the previously learned content. Eight questions were multiple choice and two questions were to be answered with free text.

Participants of the VR group were, like the noVR group, first invited to read the instructions for the ISA test and answered socio-demographic questions. During all the experiments of this group, the participants were seated on a chair wearing the HTC Vive Pro glasses. For acclimatization purposes each participant watched three initial videos. All relevant information were provided in the VR experience, which was communicated to all participants of the VR group. The VR group completed the Slater-Usoh-Steed (SUS) immersion questionnaire [26. 35] after the ISA test. The SUS is named as a reliable tool to measure presence in VR by Schwind et. al [36].

The noVR group's pretest with introduction, socio-demographic questionnaire, e-Learning and the ISA test took 32 minutes of working time. In the $V R$ group, the pretest with introduction, socio-demographic questionnaire, watching the VR video, ISA test and the SUS questionnaire took 35 minutes of working time. The VR video had a duration of 10 minutes. At the end of the experiment a qualitative feedback from all participants of the VR group was obtained through an interview. Therefore, we conducted a semi-structured interview: the aim was to respond individually to the answers of the participants using key questions and contingency questions. This interview combined open questions with requests to tell about the participants VR experience. Contingency questions were only asked if key questions were not answered satisfactorily. To check the sustainability of the acquired knowledge, both test groups had to answer the same ISA test questionnaire once again one week later without receiving the learning material again.

The data sets of repeated measurements were matched by a unique anonymous code each participant had to create at the beginning of the questionnaire in Lime Survey. Thereby, the code is generated by each subject for each measurement by providing the first and last letter of their mother's first name, their father's first 
name, their own first name, and the day of their mother's birthday (just day without month and year). A huge advantage thereby is, that the subjects do not have to remember the code but can generate it any time again and the likelihood of multiple subjects having the same code is fairly low [37].

In the ISA test a total score of 17 points could be achieved. All multiple choice questions were scored with one point. For the question with multiple choice answers a maximum score of two could be achieved if all six possible answers were clicked. With only four or five marks one point was awarded. For the free text questions there were three points for one and five points for the other.

\subsection{Learning about Social Engineering}

Social engineering was selected as the topic to be conveyed to the participants. The learning objectives of both groups were the same. Participants should learn about the term social engineering, the goals of social engineers, the characteristics of phishing, the characteristics of pretexting, the phases of a social engineering attack and ways to protect themselves from one.

The noVR group used an e-Learning platform. Content that fit the learning objectives was conveyed on several pages in the e-Learning and theoretical content was illustrated with different examples. The concept for the e-Learning platform was based on traditional learning materials. We used multiple scripts enriched with practical examples. We did not use any video material in order to determine the maximum difference in sustainability between VR and traditional e-Learning platforms. We could have used video material, but we tried to base our material on material which is often provided by small and medium-sized enterprises. However, we designed our material as interesting as possible for the participants.

The participants of the VR group watched the VR-Video. The content of the video also made sure to convey all of the learning objectives in different scenes. In the video the participants found themselves as trainees of a hacking group. The instructor made clear, that they will try to hack a company together. In the first scene an instructor explained the basics of social engineering. In the second scene the participants were brought to the headquarters of the hackers, where they learned about the phases of a social engineering attack. Together with the instructor they completed the first phase by researching personal information about a potential victim in a social network that is an employee of the target company. In the third scene the instructor and the participant were in a car in the parking area of the target company, waiting for their victim to arrive. The instructor explained the next steps: to develop trust and exploit the trust. The virtual representation of the participant approached the victim and built up trust through small talk using the information found on the social network. The participant then exploited the previously built up trust and asked the victim to give him the access code for a protected room in the company. After receiving the code the instructor and the participant used the code to gain access. During all of the scenes the instructor explained the participant, how the victim could protect himself. To not disturb the immersion when changing the scenes, a bag was pulled over the participant's head, which was removed in the subsequent scene. This should be reminiscent of movies in which criminals use this approach to obscure the way to secret places.

\subsection{Storytelling in VR}

As a narrative anchor, we have placed the user in a scenario where the user carries out a social engineering attack as the attacker. The user gets a direct practical reference with the help of this story which makes it easier to understand. In the first scene the user sees and hears the actor in front of him, who takes on the role of a social engineer of a hacking organization and speaks directly to the user (see Figure 1). The setting is intended to increase the spatial immersion. This way all senses are directed towards the actor, because the direct communication to the audience is included in the action. In addition, the scene is set up in a neutral way that the rest of the virtual environment has little to explore.

The story is plot-based and focuses on events in the story. We put plot points together meticulously in order to create a cohesive story, as the process of a typical attack is explained to the audience step by step (temporal immersion). Ulsamer et. al [38] presented an approach to navigate the viewer indoors through storytelling in VR. They evaluated highlighting of certain junctions with the help of light as the best visual method to guide the users attention. Therefore, we used visual cues to highlight necessary details. This also directs the attention to important story details, while the viewer might be distracted by long explanations and the motivation to explore the VR environment (technical immersion).

The ISA Training in VR is shot in three takes. In VR movies, the immersion of the audience is, unlike in traditional movies, negatively impaired by cuts and might lead to the viewer consciously realizing that the fictional world is not the real one. Therefore, few to no 


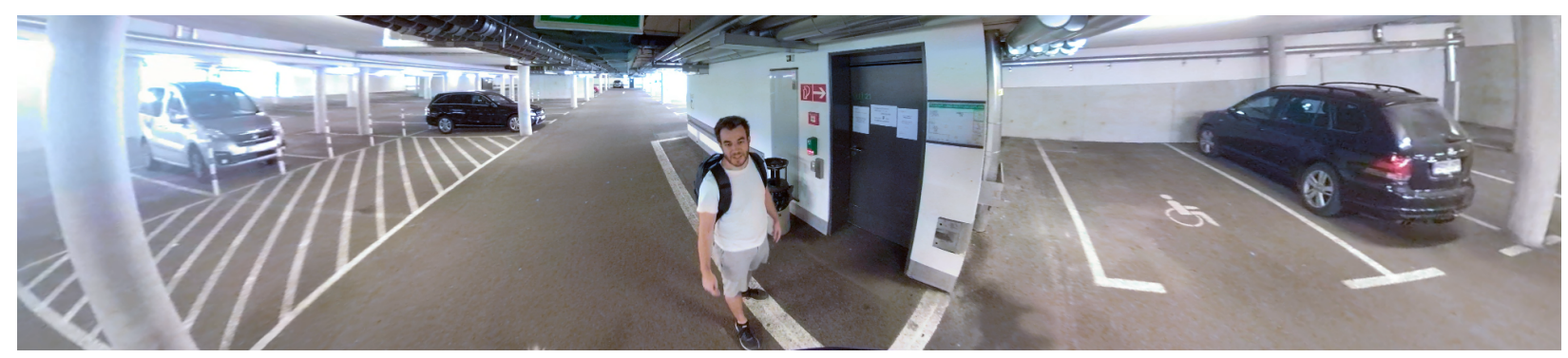

Figure 2. Creation of immersion by involving the user into the VR story.

cuts should occur in VR environments. The VR movie presented here was shot in different takes which were connected by storytelling. In order to transport the users from one place to another, a bag is pulled over their head during the story, which leaves a black image at 360 degrees. This not only allows sudden changes in the picture to be simply overplayed, but also increases emotional immersion by increasing the seriousness of the bad hacking organization.

The fact that there is little to no movement in the VR experience intends to prevent motion sickness for users. Attention guidance is achieved through actors involving the viewer. They provide the viewer with a narrative anchor as an orientation and to not to feel lost in the VR environment. In the last scene, the user is commissioned by the organization to carry out a part of the attack and to manipulate an employee of a company to steal a password for a vault. This puts the user in the emotional position to apply the previously learned techniques (see Figure2).

\subsection{Used Software, Devices, and Tools}

The surveys for the noVR and VR group were collected using the Lime Survey tool. The data was then analyzed by means of MathWork's MATLAB. The VR video was shot with the GoPro Fusion Black 360-degree action camera. Two internal cameras each record a 180-degree video, which is then stitched into a 360-degree VR video. The cinematographer attached the GoPro to a helmet and wore it for the whole recording. The camera records spatial audio in order to perceive an omnidirectional sound. For the experiment we used the full kit of the HTC Vive Pro. The initial videos for acclimatization purposes were from the SteamVR sample videos library. The software GoPro Fusion Studio 1.3 and Final Cut Pro 10.4.8 was used to edit and cut the videos. The GoPro VR Player 3.0 was used to play the finalized ISA training 360-degree video.

\section{Study Population and Sampling}

The study was conducted with a total of 50 subjects who were randomly sampled. The participants of the NoVR group $(N=25)$ were between the ages of 15 and 39. 14 participants (56\%) were male and 11 (44\%) female. Furthermore, three (12\%) participants have had prior knowledge in ISA and ten (40\%) a background in STEM. Five participants (20\%) have an academic degree, seven (28\%) a high school diploma, nine $(36 \%)$ a middle school diploma, and four (16\%) were still high school students. NoVR group's average participant was male, 24.68 years old $(\sigma=7.17)$, had no prior experience in ISA, no background in STEM, and graduated from high school. In the $V R$ group subjects were between the ages of 16 and 42.17 participants $(68 \%)$ of this group were male and eight (32\%) female $(N=25)$. Five subjects $(20 \%)$ have had prior knowledge in ISA and twelve (48\%) a background in STEM. Seven participants (28\%) have an academic degree, nine (36\%) a high school diploma, eight (32\%) a middle school diploma, and one (4\%) was still a high school student. On an average, VR participants were male, 25 years old $(\sigma=6.56)$, had no prior experience in ISA, no background in STEM, and graduated from high school.

\section{Research Results and Analysis}

As performed Kolmogorov-Smirnov tests indicated that the data was non-normally distributed, non-parametric tests were applied. To verify whether the subjects' ISA point score differed significantly between the first and second take of the ISA test, Wilcoxon's signed-rank test was applied within each group (refer to Table 1). Furthermore, to compare both point scores of noVR and VR group, the differences between the point scores were computed within each group and subsequently evaluated by means of Wilcoxon's rank-sum test (see Table 2).

All performed tests were two-tailed at the significance level of $p \leq 0.05$. Each test result 
provided the significance level $p$, an $h$-value which specifies whether the null hypothesis of equal medians can be rejected, and the $z$-value. Thereby, $h=1$ indicates that the null hypothesis of equal medians can be rejected and $h=0$ that it cannot be rejected, respectively.

\subsection{Information Security Awareness Test}

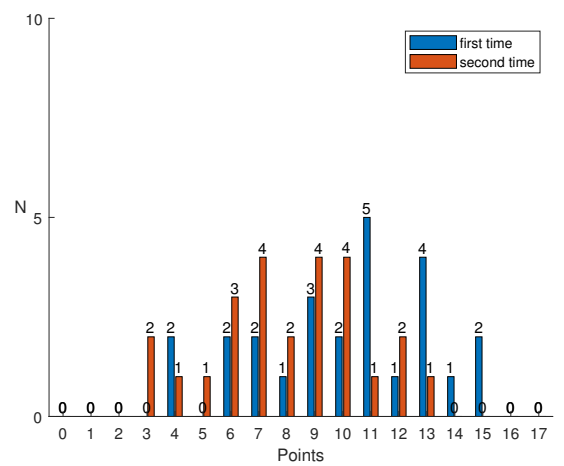

Figure 3. Score overview of the noVR group in the ISA test.

NoVR's distribution of point score at the first and second take of the ISA test are shown in Figure 3. At the first take, participants gained 10.08 points on average $(\sigma=3.16)$. In the second take, participants scored 8.04 points on average $(\sigma=2.72)$.

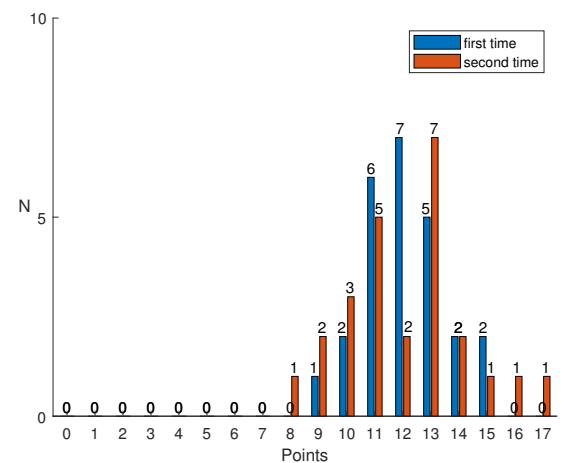

Figure 4. Score overview of the VR group in the ISA test.

Figure 4 shows VR's point score distribution of both measuring times. At the first take, subjects gained 12.08 on average $(\sigma=1.50)$. In the second take, they scored also 12.08 points on average $(\sigma=2.20)$.

A significant difference of the point score within $n o V R$ - first and second take of the ISA test - could be detected by means of Wilcoxon's signed-rank test as $p \leq 0.05$ (see Table 11. Thereby, participants gained a higher point score during the first take as the $z$-value and also the point score distribution in Figure 3 indicate.
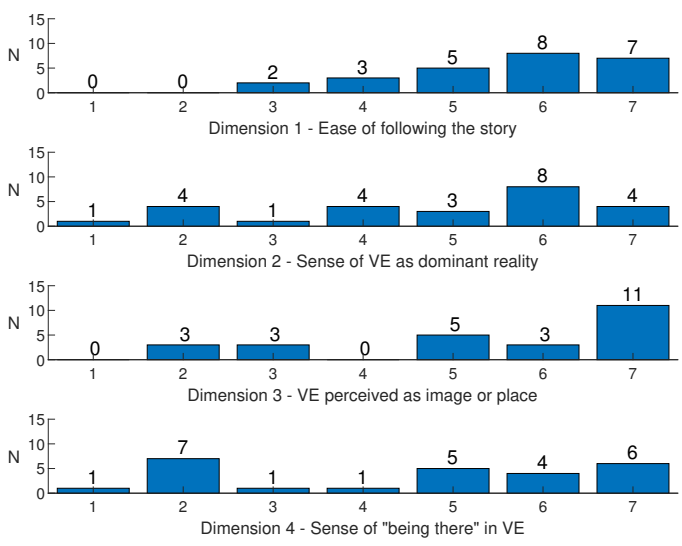

Figure 5. Results of the immersion questionnaire about the experiences in the VE.

On the other hand, there seems to be no significant difference within VR as $p>0.05$ (refer to Table 11. Surprisingly, the $z$-value and also the point score distributions shown in Figure 4 point out that subjects achieved more points during the second take of the ISA test; however, not immensely.

Table 1. Repeated measurement analysis

\begin{tabular}{|c|c|}
\hline Group & Wilcoxon Signed-Rank \\
\hline noVR & $p=0.00, h=1, z=3.54$ \\
VR & $p=0.97, h=0, z=-0.04$ \\
\hline
\end{tabular}

Comparing the point differences of the noVR and $V R$ group, the performed Wilcoxon rank-sum test indicates that there is a significant difference between both groups as $p \leq 0.05$ (see Table 2). The $z$-value as well as the point score distributions seen in Figure 3 and 4 support that the VR group achieved higher points and thereby performed better. Therefore, $H_{0}$ (see section 2 ) can be rejected and evidence supporting $H_{1}$ is found.

Table 2. Comparing noVR and VR group analysis

\begin{tabular}{|c|c|}
\hline Variable & Wilcoxon Rank-Sum \\
\hline $\begin{array}{l}\text { noVR/VR } \\
\text { difference }\end{array}$ & $p=0.00, h=1, z=-3.28$ \\
\hline
\end{tabular}

\subsection{Immersion Questionnaire}

Figure 5 shows the distribution of the immersion questionnaire. Participants rated the ease of following 
the story as 5.60 on average $(\sigma=1.26)$, on a 7-point Likert scale. The sense of the Virtual Environment (VE) as dominant reality was rated as $4.76(\sigma=1.85)$ on average. Moreover, dimension $3-\mathrm{VE}$ perceived as image or place - was rated as $5.40(\sigma=1.85)$ on average. Finally, the sense of "being there" in VE was rated as 4.52 on average $(\sigma=2.10)$.

\subsection{Qualitative Feedback}

Almost all participants stated in the qualitative interview that they were picked up from the beginning by the direct addressing of the actor and felt directly to be part of the story. Through scenes such as putting the bag over their heads, the participants became more emotionally involved in the VR experience because they felt the seriousness of the situation. They stated that this made them more concentrated in learning the content. Furthermore, the story structure made it easy to follow the individual steps of the social engineering attack. The combination of learning and direct application in the VR environment facilitated the understanding of the knowledge being conveyed.

\section{Research Discussion}

The results from our analysis tests are used to answer the research questions posed in section 2. To answer the research question RQ1 and RQ2, the analysis is taken from section 6.1 The results show that the $V R$ group performs significantly better in the ISA test than the noVR group. Therefore, we assume that an increased and sustainable knowledge in the field of social engineering can be achieved by immersive storytelling in VR as opposed to traditional e-Learnings.

In the second take of the ISA test, a significant deterioration of the participants' results from the noVR group was observed. In contrast, the score level remained approximately the same from the first to the second take of the VR group. In the second take, the participants even achieved a slightly higher score. Therefore, we also assume that immersive storytelling in VR has a more sustainable learning outcome than traditional e-Learnings have. This might be due to the sustained learning effect from the VR training.

To make assumptions about the immersion of the participants, the evaluations from section 6.2 and received qualitative feedback are utilized. Most of the participants of the $V R$ group rated the ease of following the story very high. The qualitative interview pointed out that the course of action together with the actor's guidance through the story supported the ease of following the story. Due to the spatial sound, the story could be followed while visually exploring the VE. The
"Sense of VE as dominant reality" and "Sense of being there in VE" were rated relatively even. It could be seen that some participants found it difficult to evaluate these two dimensions positively. Viewers stated in the interview that the time in the virtual environment was too short for a proper evaluation.

Very interesting were the results of Dimension 3. Nearly all participants stated that when thinking back to the VR experience they were more likely to remember the experience of being in that place rather than imagining pictures. This experience could also be seen in learning behavior. When answering the questions, participants of the VR group imagined themselves to be in the role of the social engineer in the ISA training in VR. We assume that this was one of the reasons why the VR group was able to remember the learning content better, even in the second take, than the noVR group. Despite the fact that due to the short time span of the VR video not all participants could perceive the VE as their dominant reality, they felt to be part of the story. This statement suggests that this also led to the fact that the participants not only scored better on the first ISA test, but also remembered the learning content better during the second test.

However, in order to be able to make more precise statements about the development and sustainability of knowledge in the field of social engineering, we require automated measurements. Fertig et al. mentioned the issue of divergence between knowledge and actual behavior of employees [16]. Employees often believe that they behave according to compliance. In this case, automated measurements can reveal the difference. Moreover, the measuring of knowledge by itself does not allow a truthful statement about the ISA of employees. Therefore, we have to verify if the knowledge as well as the behavior can be influenced by our VR trainings.

\section{Conclusion \& Future Work}

With the help of immersive storytelling in VR, we have carried out an experiment to increase the sustainable knowledge of ISA. The data collected from our experiment confirmed our hypothesis that not only the knowledge about ISA, but also its sustainability is much higher compared to traditional e-Learnings. It was observed that users also enjoy to explore the VR environment during the story. Since a great part of the learning content was conveyed audibly, it was difficult for the participants not to notice important content. However, the VR video could not be paused to reflect on what they had learned. It was also not possible to 
interact with the actors which could have prolonged the sustainable effect of this ISA training.

Considering these theories, we would like to use the concept of anchored instructions in future work to present the user with a problem that should be solved by the user [39]. In addition, the user should have the opportunity to interact with digital characters and make decisions that influence the progress of the story. Rothe et. al [40] wrote a taxonomy that qualified and quantified different methods for attention guidance in cinematic virtual reality. We want to use different methods of attention guidance to provide the user with as much learning content as possible without causing immersion losses.

As we only used a traditional e-Learning platform we could not compare the differences in sustainability between VR video and traditional video material. For this reason, we will conduct a second study in which we will create cartoon video material which will use the same storyline as our VR videos do. We will then determine if videos by itself will increase the sustainability or if the greater immersion of VR videos will increase the sustainability as well.

Since we solely measured the participants knowledge, we cannot make any assumptions about the actual ISA as well as the actual behavior of our participants. Therefore, we have to conduct additional studies to verify if VR training can also improve the overall ISA. Before doing so, there are some challenges which have to be solved: how to measure the behavior of randomly picked participants and how to consider privacy aspects when doing the measurements. Future work will test whether VR can influence other factors such as salience, habit or intention. Since each individuals behavior is also formed by experiences from the past [41], it would be particularly interesting to find out whether VR is capable of creating such experiences.

\section{References}

[1] ISACA, "State of Cybersecurity 2017. Part 2: Current Trends in Threat Landscape," tech. rep., Information Systems Audit and Control Association, 2017. Published: ISACA, 3701 Algonquin Road, Suite 1010 Rolling Meadows, IL 60008 USA.

[2] K. D. Mitnick and W. L. Simon, The Art of Deception: Controlling the Human Element of Security. New York, NY, USA: John Wiley \& Sons, Inc., 2002.

[3] T. Gundu, S. Flowerday, and K. Renaud, "Deliver Security Awareness Training, then Repeat: Deliver; Measure Efficacy," in 2019 Conference on Information Communications Technology and Society (ICTAS), pp. 1-6, Mar. 2019.

[4] V. Román-Ibáñez, F. A. Pujol-López, H. Mora-Mora, M. L. Pertegal-Felices, and A. Jimeno-Morenilla, "A Low-Cost Immersive Virtual Reality System for Teaching Robotic Manipulators Programming," Sustainability, vol. 10, p. 1102, Apr. 2018. Number: 4 Publisher: Multidisciplinary Digital Publishing Institute.

[5] L. Freina and M. Ott, "A Literature Review on Immersive Virtual Reality in Education: State of the Art and Perspectives," in Conference Proceedings of eLearning and Software for Education (eLSE), pp. 133-141, 2015.

[6] Y. Liu, "Virtual neurosurgical education for image-guided deep brain stimulation neurosurgery," in 2014 International Conference on Audio, Language and Image Processing, July 2014.

[7] S. Rizvic, D. Boskovic, F. Bruno, B. D. Petriaggi, S. Sljivo, and M. Cozza, "Actors in VR storytelling," in 2019 11th International Conference on Virtual Worlds and Games for Serious Applications (VS-Games), pp. 1-8, Sept. 2019. ISSN: 2474-0489.

[8] B. B. Gupta, N. A. G. Arachchilage, and K. E. Psannis, "Defending against phishing attacks: taxonomy of methods, current issues and future directions," Telecommunication Systems, vol. 67, pp. 247-267, Feb. 2018.

[9] C. Hadnagy and P. Wilson, Social Engineering: The Art of Human Hacking. Wiley, 2011.

[10] K. Ivaturi and L. Janczewski, "A taxonomy for social engineering attacks," in International Conference on Information Resources Management, pp. 1-12, Centre for Information Technology, Organizations, and People, 2011.

[11] P. Tetri and J. Vuorinen, "Dissecting social engineering," Behaviour \& Information Technology, vol. 32, no. 10, pp. 1014-1023, 2013.

[12] B. Semba and T. Eymann, "Developing a Model to Analyze the Influence of Personal Values on IT Security Behavior.," in Tagungsband Multikonferenz. Wirtschaftsinformatik 2016, (Ilmenau), pp. 1083 - 1091, TU Ilmenau, 2016.

[13] L. Hirshfield, P. Bobko, A. J. Barelka, M. R. Costa, G. J. Funke, V. F. Mancuso, V. Finomore, and B. A. Knott, "The Role of Human Operators' Suspicion in the Detection of Cyber Attacks:," International Journal of Cyber Warfare and Terrorism, vol. 5, pp. 28-44, July 2015.

[14] A. E. Schütz, "Information Security Awareness: Its Time to Change Minds!," in Proceedings of International Conference on Applied Informatics Imagination, Creativity, Design, Development - ICDD 2018, (Sibiu, Romania), 2018.

[15] A. E. Schütz, T. Fertig, K. Weber, and N. H. Müller, "How E-Learning Can Facilitate Information Security Awareness," in Learning and Collaboration Technologies. Designing Learning Experiences (P. Zaphiris and A. Ioannou, eds.), (Cham), pp. 390-401, Springer International Publishing, 2019.

[16] T. Fertig, A. E. Schütz, K. Weber, and N. H. Müller, "Measuring the Impact of E-Learning Platforms on Information Security Awareness," in Learning and Collaboration Technologies. Designing Learning Experiences (P. Zaphiris and A. Ioannou, eds.), Lecture Notes in Computer Science, (Cham), pp. 26-37, Springer International Publishing, 2019.

[17] W. Hsu, H. K. Lin, and Y. Lin, "The research of applying Mobile Virtual Reality to Martial Arts learning system with flipped classroom," in 2017 International Conference on Applied System Innovation (ICASI), pp. 1568-1571, 2017. 
[18] F. Yang and W. Wu, "The Application of Virtual Reality in E-Learning," in 2010 International Conference on E-Business and E-Government, pp. 5548-5551, 2010.

[19] J. Saunier, M. Barange, B. Blandin, R. Querrec, and J. Taoum, "Designing Adaptable Virtual Reality Learning Environments," in Proceedings of the 2016 Virtual Reality International Conference, VRIC 16, (New York, NY, USA), Association for Computing Machinery, 2016.

[20] P. Danilicheva, S. Klimenko, Y. Baturin, and A. Serebrov, "Education in Virtual Worlds: Virtual Storytelling," in 2009 International Conference on CyberWorlds, pp. 333-338, 2009.

[21] A. Tse, C. Jennett, J. Moore, Z. Watson, J. Rigby, and A. L. Cox, "Was i there? impact of platform and headphones on 360 video immersion," in Proceedings of the 2017 CHI Conference Extended Abstracts on Human Factors in Computing Systems, CHI EA '17, (New York, NY, USA), p. 29672974, Association for Computing Machinery, 2017.

[22] A. MacQuarrie and A. Steed, "Cinematic virtual reality: Evaluating the effect of display type on the viewing experience for panoramic video," in 2017 IEEE Virtual Reality (VR), pp. 45-54, 2017.

[23] H.-M. Huang, U. Rauch, and S.-S. Liaw, "Investigating learners attitudes toward virtual reality learning environments: Based on a constructivist approach," Computers \& Education, vol. 55, no. 3, pp. 1171 - 1182, 2010.

[24] A. Sutcliffe, Multimedia and Virtual Reality: Designing Usable Multisensory User Interfaces. USA: L. Erlbaum Associates Inc., 2002.

[25] A. Elmezeny, N. Edenhofer, and J. Wimmer, "Immersive Storytelling in 360-Degree Videos: An Analysis of Interplay Between Narrative and Technical Immersion," Journal of Virtual Worlds Research, vol. 11, 042018.

[26] M. Slater and M. Usoh, "Body Centred Interaction in Immersive Virtual Environments," in Artificial Life and Virtual Reality, pp. 125-148, John Wiley and Sons, 1994.

[27] A. Sheikh, A. Brown, Z. Watson, and M. Evans, "Directing attention in 360-degree video," IET Conference Proceedings, 2016.

[28] S. Rothe, H. Hußmann, and M. Allary, "Diegetic Cues for Guiding the Viewer in Cinematic Virtual Reality," in Proceedings of the 23rd ACM Symposium on Virtual Reality Software and Technology, VRST 17, (New York, NY, USA), Association for Computing Machinery, 2017.

[29] L. T. Nielsen, M. B. Møller, S. D. Hartmeyer, T. C. M. Ljung, N. C. Nilsson, R. Nordahl, and S. Serafin, "Missing the Point: An Exploration of How to Guide Users Attention during Cinematic Virtual Reality," in Proceedings of the 22nd ACM Conference on Virtual Reality Software and Technology, VRST 16, (New York, NY, USA), p. 229232, Association for Computing Machinery, 2016.

[30] R. Pausch, J. Snoddy, R. Taylor, S. Watson, and E. Haseltine, "Disneys Aladdin: First Steps toward Storytelling in Virtual Reality," in Proceedings of the 23rd Annual Conference on Computer Graphics and Interactive Techniques, SIGGRAPH 96, (New York, NY, USA), p. 193203, Association for Computing Machinery, 1996.
[31] C. Brown, G. Bhutra, M. Suhail, Q. Xu, and E. D. Ragan, "Coordinating attention and cooperation in multi-user virtual reality narratives," in 2017 IEEE Virtual Reality (VR), pp. 377-378, 2017.

[32] A. Silva, G. Raimundo, and A. Paiva, "Tell Me That Bit Again... Bringing Interactivity to a Virtual Storyteller," in Virtual Storytelling. Using Virtual Reality Technologies for Storytelling (O. Balet, G. Subsol, and P. Torguet, eds.), (Berlin, Heidelberg), pp. 146-154, Springer Berlin Heidelberg, 2003.

[33] M.-L. Ryan, Narrative as Virtual Reality 2: Revisiting Immersion and Interactivity in Literature and Electronic Media., vol. 2. Baltimore : Johns Hopkins University Press, 2015.

[34] Y. Cai, C. Miao, A.-H. Tan, and Z. Shen, "A Hybrid of Plot-Based and Character-Based Interactive Storytelling," in Technologies for E-Learning and Digital Entertainment (K.-c. Hui, Z. Pan, R. C.-k. Chung, C. C. L. Wang, X. Jin, S. Göbel, and E. C.-L. Li, eds.), (Berlin, Heidelberg), pp. 260-273, Springer Berlin Heidelberg, 2007.

[35] M. Usoh, E. Catena, S. Arman, and M. Slater, "Using Presence Questionnaires in Reality," Presence: Teleoperators and Virtual Environments, vol. 9, pp. 497-503, 042000.

[36] V. Schwind, P. Knierim, N. Haas, and N. Henze, "Using Presence Questionnaires in Virtual Reality," in Proceedings of the 2019 CHI Conference on Human Factors in Computing Systems, CHI 19, (New York, NY, USA), p. 112, Association for Computing Machinery, 2019.

[37] University of Giessen, "Code für Panelstudien." https://www.uni-giessen.de/org/admin/ stab/stl/servicestelle/panelcode. Last Visited - 072020.

[38] P. Ulsamer, K. Pfeffel, and N. H. Müller, "Indoor Navigation Through Storytelling in Virtual Reality," in Learning and Collaboration Technologies. Ubiquitous and Virtual Environments for Learning and Collaboration (P. Zaphiris and A. Ioannou, eds.), (Cham), pp. 230-239, Springer International Publishing, 2019.

[39] L. Li, H. Mao, and L. Xu, "Application of concept maps-based anchored instruction in programming course," in 2010 10th IEEE International Conference on Computer and Information Technology, pp. 2196-2200, 2010 .

[40] S. Rothe, D. Buschek, and H. Hussmann, "Guidance in Cinematic Virtual Reality-Taxonomy, Research Status and Challenges," Multimodal Technologies and Interaction, vol. 3, p. 19, 032019.

[41] D. E. Montaño and D. Kasprzyk, "Theory of Reasoned Action, Theory of Planned Behavior, and the Integrated Behavior Model," in Health Behavior and Health Education (K. Glanz, Rimer, Barbara, K., and K. Viswanath, eds.), pp. 67-96, APA PsycNet, 2008. 